\title{
Utilização da tecnologia de freqüência duplicada em pacientes submetidos a "laser assisted in situ keratomileusis"(LASIK): Análise de custo-benefício
}

\author{
Frequencydoubling technology in patients submitted to laser-assisted in situ \\ keratomileusis(LASIK):Cost-benefit analysis
}

Fernanda Bon Duarte ${ }^{1}$

Rejane Carvalho Aires ${ }^{2}$

Fernando Mutton ${ }^{3}$

Augusto Paranhos Jr. ${ }^{4}$

\section{RESUMO}

Objetivo: Verificar existência de alteração funcional das células magnocelulares por meio da tecnologia de freqüência duplicada (FDT), após o incremento de pressão gerado pela técnica de "laser assisted in situ keratomileusis" (LASIK), e avaliar os benefícios de realizar este em pacientes candidatos à cirurgia refrativa, considerando-se os custos envolvidos nos procedimentos. Métodos: Estudo prospectivo em 19 pacientes com ametropias, submetidos à cirurgia "laser assisted in situ keratomileusis", no Hospital Oftalmológico de Sorocaba. Foram realizados 2 exames de tecnologia de freqüência duplicada em ambos os olhos dos pacientes antes da cirurgia por "laser assisted in situ keratomileusis", e um exame após a cirurgia. A avaliação da diferença entre as medidas de MD ("mean deviation") e PSD ("pattern standard deviation") efetuadas pela tecnologia de freqüência duplicada antes e depois da cirurgia refrativa foram avaliadas por meio do teste de t pareado. A diferença entre as medidas de MD (Delta MD) foi considerada variável dependente para elaborações de uma avaliação de regressão múltipla. Foi realizada uma análise de custos do exame de tecnologia de freqüência duplicada e da cirurgia "laser assisted in situ keratomileusis". Resultados: Não houve diferença entre as medidas de MD no exame de tecnologia de freqüência duplicada antes e após o procedimento $(p=0,4454)$ assim como para PSD $(p=0,9716)$. Nenhuma variável contínua testada influiu significantemente no Delta $\mathrm{MD}\left(\mathrm{r}^{2}=4,1 \% ; \mathrm{F}=0,15\right.$; $\mathrm{p}=0,960$ ). Considerando a realização de 1500 cirurgias de "laser assisted in situ keratomileusis" ao ano no Hospital Oftalmológico de Sorocaba, o custo médio desta cirurgia é de R\$373,27 e o custo do exame de tecnologia de freqüência duplicada é de R\$13,15 por paciente. Conclusões: Não houve diferença estatisticamente significante entre as medidas de MD no exame de tecnologia de freqüência duplicada antes e após o procedimento assim como para PSD. A tecnologia de freqüência duplicada, exame com alta sensibilidade e especificidade, e de baixo custo poderia ser utilizado como exame pré-operatório em pacientes submetidos à cirurgia refrativa (LASIK), de alto custo, com o propósito de evitar tal procedimento em pacientes com glaucoma.

Descritores: Ceratomileuse assistida por excimer laser in situ; Análise custo-benefício; Erros de refração/cirurgia; Células ganglionares da retina/patologia; Perimetria/métodos

Nota Editorial: Pela análise deste trabalho e por sua anuência na divulgação desta nota, agradecemos à Dra. Christiane Rolim de Moura. 


\section{INTRODUÇ̃̃̃O}

Técnicas para avaliar campo visual existem há muitos anos. Desde a mais simples e grosseira como o campo de confrontação, até as mais atuais, dentre as quais podemos citar a tecnologia de freqüência duplicada (FDT) $)^{(1)}$.

Até hoje, a perimetria branco no branco tem sido considerada como "padrão ouro" para detecção de perdas no campo visual $^{(2-5)}$. Entretanto é necessária uma lesão de pelo menos $50 \%$ dos axônios das células ganglionares da retina para que se inicie alguma alteração neste exame ${ }^{(6-7)}$.

Com o propósito de se detectar mais precocemente a perda do campo visual surgiu o FDT. As células ganglionares da retina são compostas pelas células magnocelulares que correspondem a aproximadamente $15 \%$ do total. A tecnologia de freqüência duplicada estimula apenas um subgrupo de células magnocelulares, as células My que respondem a estímulos de alta freqüência temporal $(25 \mathrm{~Hz})$ e baixa freqüência espacial $(0,25 \text { ciclos/grau })^{(8)}$.

Durante a confecção do "flap" corneano em cirurgias refrativas "laser assisted in situ keratomileusis" (LASIK) o anel de sucção impõe ao olho pressões maiores do que $65 \mathrm{mmHg}^{(9)}$. Alguns autores relatam isquemia de nervo óptico e perda de campo visual em paciente suspeito de glaucoma submetido a LASIK $^{(10)}$. Há a descrição de um caso de neuropatia óptica bilateral manifestada por alterações de campo visual, baixa de acuidade visual, alterações da visão de cores, aumento de escavação, alterações da camada de fibras nervosas da retina e defeitos da rima neural após LASIK ${ }^{(11)}$.

Alguns autores mostram que há diferença estatisticamente significante entre a espessura da camada de fibras nervosas da retina medida pela polarimetria de varredura a laser (GDX) antes e após LASIK, porém não atribuem esta diferença a um dano anatômico e sim à alteração da bi-refringência corneana ${ }^{(12)}$. Outros não mostram diferença entre as medidas da espessura da camada de fibras nervosas da retina antes e após o LASIK, porém, a sua amostra apresenta menor equivalente esférico e, portanto menor ablação alterando menos o poder de birrefringência corneana ${ }^{(13)}$. Ao comparar três técnicas de avaliação de dano anatômico: GDx, tomografia de varredura a laser e tomografia de coerência óptica, sendo que os dois últimos não são influenciados por alterações na polarização corneana, GursesOzden et al comprovaram ausência de dano endossando que a alteração que ocorre na leitura do GDx se deve a alteração na estrutura corneana induzida pelo procedimento ${ }^{(14)}$.

Os objetivos deste trabalho são verificar se existe alteração funcional das células ganglionares magnocelulares, por meio da tecnologia de freqüência duplicada (FDT), após o incremento de pressão gerado pela técnica LASIK para cirurgia refrativa, tendo-se em vista a alta sensibilidade do FDT para detectar dano inicial glaucomatoso ${ }^{(15-16)}$ e avaliar os benefícios de realizar o exame de FDT em pacientes candidatos a cirurgia refrativa, levando-se em consideração os custos envolvidos nos procedimentos.

\section{MÉTODOS}

O projeto de pesquisa foi aprovado pelo Comitê de Ética Médica do Hospital Oftalmológico de Sorocaba.

Estudo prospectivo em pacientes apresentando biomicroscopia, tonometria e fundoscopia normais e apresentando qualquer ametropia, que se submeteriam à cirurgia refrativa (LASIK) no Hospital Oftalmológico de Sorocaba no período de maio de 2001 a março de 2002.

Foram realizados 3 exames de tecnologia de freqüência duplicada (FDT-Zeiss Humphrey Systems) em ambos os olhos de paciente que seriam submetidos à cirurgia refrativa Laser Assisted in situ Keratomileusis (LASIK)

Os exames dividiram-se do seguinte modo: 2 exames antes da cirurgia refrativa sendo o primeiro com a estratégia "screening" e o segundo com a estratégia "full threshold N-30" e 1 exame com a estratégia "full threshold N-30" após a cirurgia.

Todos os exames realizados antes da cirurgia foram feitos com a luz ambiente apagada e com o uso da correção e sempre após um termo de consentimento livre e assistido assinado pelo paciente.

Os exames realizados após a cirurgia foram feitos também com a luz ambiente apagada e sem uso de correção e num intervalo de 24 a 32 horas após a mesma.

Escolha do olho: Para efeito de cálculo, quando dois olhos eram elegíveis, o olho de menor refração esférica era escolhido, por desejarmos avaliar o efeito do procedimento em si (pressão elevada durante a cirurgia) e não influência de grandes vícios de refração. Quando a refração esférica pré-cirurgia era igual nos dois olhos, foi efetuada randomização do olho.

A avaliação da diferença entre as medidas de MD (mean deviation) e PSD (pattern mean deviation) efetuadas pelo FDT antes e depois da cirurgia refrativa foram avaliadas por meio do teste de T pareado. A diferença entre as medidas de MD (Delta MD) foi considerada variável dependente para elaborações de uma avaliação de regressão múltipla tendo como variáveis preditoras as seguintes: idade, paquimetria, ablação, refração esférica antes do procedimento. A avaliação de colinearidade foi executada por meio do teste de VIF (fator de inflação de variância) e a exeqüibilidade por meio de teste de homocedasticidade (que foi satisfeito).

Os custos envolvidos na cirurgia refrativa e com o exame de FDT foram levantados no Hospital Oftalmológico de Sorocaba.

\section{RESULTADOS}

A amostra constou de 19 olhos de 19 pacientes sendo 12 do sexo masculino $(63,16 \%)$ e 7 do sexo feminino (36,84\%). Os demais dados de estatística descritiva se encontram na tabela 1.

Não houve diferença entre as medidas de MD no FDT antes e após o procedimento $(\mathrm{p}=0,4454)$ (Tabela 2) assim como para PSD ( $p=0,9716)$ (Tabela 3). Nenhuma variável contínua testada influiu de forma significante o Delta MD $\left(\mathrm{r}^{2}=\right.$ 4,1\%; F= 0,15; $\mathrm{p}=0,960)$ (Tabela 4). 


\begin{tabular}{|c|c|c|c|c|c|c|c|c|}
\hline & $\mathbf{N}$ & Média & DP & Mediana & Mínimo & Máximo & $\begin{array}{l}\text { Quartil } \\
\text { inferior }\end{array}$ & $\begin{array}{l}\text { Quartil } \\
\text { superior }\end{array}$ \\
\hline Idade & 19 & 31,4737 & 7,69826 & 32,0000 & 22,0000 & 46,0000 & 24,0000 & 38,0000 \\
\hline Paquimetria & 19 & 546,2105 & 30,12857 & 535,0000 & 504,0000 & 610,0000 & 525,0000 & 566,0000 \\
\hline Ablação & 19 & 58,8842 & 32,76733 & 70,7000 & 8,4000 & 125,4000 & 31,0000 & 83,3000 \\
\hline Rx esf pré & 19 & $-3,6053$ & 3,69150 & $-4,7500$ & $-12,0000$ & 5,5000 & $-6,0000$ & $-1,2500$ \\
\hline Rx esf pós & 19 & $-0,2763$ & 0,50618 & 0,0000 & $-1,5000$ & 0,2500 & $-0,5000$ & 0,0000 \\
\hline Equi pré & 19 & $-4,2211$ & 3,43531 & $-5,5000$ & $-12,0000$ & 2,2500 & $-6,5000$ & $-1,5000$ \\
\hline Equi pós & 19 & $-0,3289$ & 0,53394 & 0,0000 & $-1,7500$ & 0,2500 & $-0,5000$ & 0,0000 \\
\hline MD pré & 19 & $-1,3932$ & 2,33320 & $-0,6900$ & $-5,8700$ & 2,0400 & $-3,4900$ & 0,6100 \\
\hline MD pós & 19 & $-1,0726$ & 2,21903 & $-0,6600$ & $-5,2200$ & 2,2200 & $-3,1800$ & 0,9600 \\
\hline MD delta & 19 & 0,3205 & 1,79066 & 0,3900 & $-3,2000$ & 4,2000 & $-0,5700$ & 0,6600 \\
\hline PSD pré & 19 & 4,0447 & 1,03737 & 3,7900 & 2,7100 & 6,4600 & 3,2500 & 4,2400 \\
\hline PSD pós & 19 & 4,0526 & 1,18189 & 3,7800 & 2,4700 & 6,5500 & 2,9700 & 4,8400 \\
\hline PSD delta & 19 & 0,0079 & 0,95659 & $-0,1600$ & $-1,4600$ & 1,9400 & $-0,7700$ & 0,9200 \\
\hline
\end{tabular}

\begin{tabular}{|lccccccc|}
\hline \multicolumn{7}{|c|}{ Tabela 2. Avaliação da diferença entre as medidas de MD antes e depois do procedimento } \\
MD pré & $\mathbf{N}$ & Média & DP & Diferença & DP Dif & t & df \\
MD pós & 19 & $-1,39316$ & 2,333198 & & & & \\
MD= mean deviation; $M D$ & $-1,07263$ & 2,219033 & $-0,320526$ & 1,790662 & $-0,780237$ & 18 & 0,445391 \\
\hline
\end{tabular}

\begin{tabular}{|c|c|c|c|c|c|c|c|c|}
\hline & $\mathbf{N}$ & Média & DP & Diferença & DP Dif & $t$ & $d f$ & $\mathbf{P}$ \\
\hline PSD pré & & 4,044737 & 1,037365 & & & & & \\
\hline PSD pós & 19 & 4,052632 & 1,181886 & $-0,007895$ & 0,956589 & $-0,035974$ & 18 & 0,971699 \\
\hline
\end{tabular}

\begin{tabular}{|c|c|c|c|c|c|}
\hline & & & & & \\
\hline Fator preditor & Coeficiente & DP & t & $\mathbf{p}$ & VIF \\
\hline Constante & $-4,182$ & 8,695 & $-0,48$ & 0,638 & \\
\hline Idade & $-0,01224$ & 0,06316 & $-0,19$ & 0,849 & 1,1 \\
\hline Paquimetria & 0,00825 & 0,01620 & 0,51 & 0,619 & 1,1 \\
\hline Ablação & 0,00394 & 0,02407 & 0,16 & 0,872 & 2,8 \\
\hline Refração esférica pré & $-0,0418$ & 0,2142 & $-0,20$ & 0,848 & 2,8 \\
\hline
\end{tabular}

Os custos fixos e variáveis relacionados à cirurgia refrativa LASIK do Hospital Oftalmológico de Sorocaba estão representados nas tabelas 5 e 6 respectivamente:

Temos, portanto, o valor de R\$229.400,00 ao ano de depreciação dos equipamentos, que somado aos outros itens de custo fixo como manutenção e energia totalizam o valor de R\$ 327.400,00 ao ano. O custo variável por paciente (subentendendo que o mesmo opera ambos os olhos) é de $\mathrm{R} \$ 155,00$.

\begin{tabular}{|c|c|c|c|}
\hline & & Valor & Depreciação \\
\hline Excimer laser & $\mathrm{R} \$$ & $1.000 .000,00$ & $\mathrm{R} \$ 200.000,00$ ano \\
\hline Microcerátomo & $\mathrm{R} \$$ & $140.000,00$ & $\mathrm{R} \$ \quad 28.000,00$ ano \\
\hline Autoclave & $\mathrm{R} \$$ & $7.000,00$ & $\mathrm{R} \$ \quad 1.400,00$ ano \\
\hline Manutenção & $\mathrm{R} \$$ & $92.000,00$ ano & \\
\hline Energia & $\mathrm{R} \$$ & $6.000,00$ ano & \\
\hline Total & $\mathrm{R} \$$ & $1.245 .000,00$ & $\mathrm{R} \$ 229.400,00$ ano \\
\hline
\end{tabular}


Consideramos que 1.500 cirurgias são realizadas ao ano no Hospital Oftalmológico de Sorocaba e fizemos uma estimativa para completar a tabela 7 na qual calculamos o custo médio da cirurgia por paciente.

Considerando a realização de 1.500 exames de FDT ao ano, custo fixo de depreciação de $\mathrm{R} \$ 3.600,00$ ao ano e custo variável por exame de $\mathrm{R} \$ 10,75$, podemos calcular o custo médio de cada exame. O custo médio é a somatória do custo fixo por paciente $(\mathrm{R} \$ 2,4)$ com o custo variável $(\mathrm{R} \$ 10,75)$ que é, portanto de $\mathrm{R} \$ 13,15$.

\begin{tabular}{|c|c|c|c|}
\hline Material & $\begin{array}{c}\text { Valor } \\
\text { unitário }\end{array}$ & $\begin{array}{l}\text { Quantidade } \\
\text { por paciente }\end{array}$ & $\begin{array}{l}\text { Custo final } \\
\text { por paciente }\end{array}$ \\
\hline Colírio anestésico & $\mathrm{R} \$ 4,56$ & $1 / 5$ & $\mathrm{R} \$ 1,00$ \\
\hline Colírio Tobrex ${ }^{\circledR}$ & $\mathrm{R} \$ 12,17$ & $1 / 5$ & $R \$ 2,50$ \\
\hline Colírio Acular ${ }^{\circledR}$ & $\mathrm{R} \$ 28,89$ & $1 / 5$ & $\mathrm{R} \$ 5,80$ \\
\hline Sol BSS ${ }^{\circledR}-15 \mathrm{ml}$ & $\mathrm{R} \$ 20,60$ & $1 / 2$ & $\mathrm{R} \$ 10,30$ \\
\hline Campo & $\mathrm{R} \$ 0,20$ & 1 & $R \$ 0,20$ \\
\hline Compressa & $\mathrm{R} \$ 0,10$ & 1 & $\mathrm{R} \$ 0,10$ \\
\hline Máscara & $\mathrm{R} \$ 0,12$ & 3 & $\mathrm{R} \$ 0,36$ \\
\hline Gorro & $\mathrm{R} \$ 0,10$ & 3 & $\mathrm{R} \$ 0,30$ \\
\hline Turbante & $\mathrm{R} \$ 0,10$ & 1 & $\mathrm{R} \$ 0,10$ \\
\hline Avental & $R \$ 1,00$ & 3 & $\mathrm{R} \$ 3,00$ \\
\hline Propé & $\mathrm{R} \$ 0,10$ & 3 & $\mathrm{R} \$ 0,30$ \\
\hline Micropore & $\mathrm{R} \$ 3,00$ & $1 / 20$ & $\mathrm{R} \$ 0,15$ \\
\hline Gaze estéril & $\mathrm{R} \$ 0,15$ & 1 & $\mathrm{R} \$ 0,15$ \\
\hline Campo Visitek & $R \$ 2,80$ & 3 & $\mathrm{R} \$ 8,40$ \\
\hline Merocel & $\mathrm{R} \$ 23,61$ & 1 & $\mathrm{R} \$ 23,61$ \\
\hline Cânula de irrigação & $R \$ 15,45$ & $1 / 5$ & $R \$ 3,09$ \\
\hline Lâmina Hansatome & $\mathrm{R} \$ 119,70$ & $1 / 2$ & $\mathrm{R} \$ 59,85$ \\
\hline Filtro $\mathrm{c} /$ mangueira & $\mathrm{R} \$ 37,70$ & $1 / 50$ & $R \$ 0,75$ \\
\hline Gás premix & $R \$ 2.600,00$ & $1 / 200$ & $\mathrm{R} \$ 13,00$ \\
\hline Gás N2 & $\mathrm{R} \$ 1.417,00$ & $1 / 300$ & $\mathrm{R} \$ 4,73$ \\
\hline Gás Hélio & $\mathrm{R} \$ 1.400,00$ & $1 / 10.000$ & $\mathrm{R} \$ 0,14$ \\
\hline Funcionários & & & $\mathrm{R} \$ 15,00$ \\
\hline Esterilização & & & $\mathrm{R} \$ 2,00$ \\
\hline Povidine & $\mathrm{R} \$ 1,78$ & $1 / 10$ & $\mathrm{R} \$ 0,17$ \\
\hline Total & & & $R \$ 155,00$ \\
\hline
\end{tabular}

\section{DISCUSS ÃO}

Entende-se por custos variáveis aqueles que estão diretamente relacionados com o volume de cirurgias. Quanto maior o volume de cirurgia, maior o total do custo variável, entretanto, o custo variável é fixo para cada paciente ${ }^{(17)}$.

Entende-se por custos fixos, aqueles que não estão relacionados ao volume de atendimento, eles estão estáveis. Estes custos, porém, influenciam diretamente sobre o custo médio visto que quanto maior o volume de atendimento, maior é o "rateio" destes custos fixos e menor o custo médio. Portanto, para um bom gerenciamento a maior preocupação é focada nos custos fixos ${ }^{(17)}$.

Deve-se ter em mente que considerar como custo de cirurgia refrativa apenas o valor “custo variável” é ilusório. Temos que incluir todo o investimento realizado e a depreciação do equipamento. Assim, se forem consideradas 1.500 cirurgias refrativas (LASIK) ao ano o custo médio de cirurgias por paciente seria de $\mathrm{R} \$ 373,27$.

Analisando os custos do exame de tecnologia de freqüência duplicada podemos notar que é baixo $(\mathrm{R} \$ 13,15)$, pois se trata de um equipamento de custos fixo e variável pequenos.

Sabe-se que a pressão ocular não é um bom método para "screening" de glaucoma, visto que identifica menos da metade dos pacientes com a doença ${ }^{(18)}$. Outros métodos para detecção precoce de glaucoma existem, como o OCT (Optical Coherence Tomography) e SWAP (Short Wavelenght Automated Perimetry), entretanto apresentam maior custo em relação ao FDT. Bowd et al $^{(19)}$ mostram que o OCT apresenta maior sensibilidade (70-80\%) seguido pelo FDT (70-80\%) e após pelo SWAP (40$52 \%)$. Seria necessário, portanto um método de alta sensibilidade e especificidade e de baixo custo que pudesse identificar pacientes com alterações, que contra-indicassem um procedimento de alto custo como a cirurgia refrativa.

Os estudos encontrados na literatura nos mostram não haver dano anatômico na camada de fibras nervosas imposto pelo aumento de pressão gerado pelo $\operatorname{LASIK}^{(12-14)}$ e até o presente momento não conseguimos encontrar nenhuma publicação que avaliasse o dano funcional das células ganglionares. Se ocorresse o dano funcional, ele seria leve, e consequentemente

\begin{tabular}{|c|c|c|c|c|c|c|}
\hline $\begin{array}{l}\text { Número de } \\
\text { cirurgias }\end{array}$ & $\begin{array}{l}\text { Custo fixo } \\
\text { depreciação }\end{array}$ & $\begin{array}{l}\text { Custo variável } \\
\text { por cirurgia }\end{array}$ & $\begin{array}{c}\text { Custo total } \\
\text { variável }\end{array}$ & $\begin{array}{l}\text { Custo } \\
\text { total }\end{array}$ & $\begin{array}{l}\text { Custo fixo } \\
\text { por cirurgia }\end{array}$ & $\begin{array}{l}\text { Custo } \\
\text { médio }\end{array}$ \\
\hline 700 & $327.400,00$ & 155,00 & $108.500,00$ & $435.900,00$ & 467,71 & 622,71 \\
\hline 810 & $327.400,00$ & 155,00 & $125.550,00$ & $452.950,00$ & 404,20 & 559,20 \\
\hline 900 & $327.400,00$ & 155,00 & $139.500,00$ & $466.900,00$ & 363,78 & 518,78 \\
\hline 1020 & $327.400,00$ & 155,00 & $158.100,00$ & $485.500,00$ & 320,98 & 475,98 \\
\hline 1200 & $327.400,00$ & 155,00 & $186.000,00$ & $513.400,00$ & 272,83 & 427,83 \\
\hline 1320 & $327.400,00$ & 155,00 & $204.600,00$ & $532.000,00$ & 248,03 & 403,03 \\
\hline 1500 & $327.400,00$ & 155,00 & $232.500,00$ & $559.900,00$ & 218,27 & 373,27 \\
\hline 1700 & $327.400,00$ & 155,00 & $263.500,00$ & $590.900,00$ & 192,59 & 347,59 \\
\hline 1870 & $327.400,00$ & 155,00 & $289.850,00$ & $617.250,00$ & 175,08 & 330,08 \\
\hline 2000 & $327.400,00$ & 155,00 & $310.000,00$ & $637.400,00$ & 163,70 & 318,70 \\
\hline
\end{tabular}




\begin{tabular}{|c|c|c|}
\hline & Valor & Depreciação \\
\hline FDP & $\mathrm{R} \$ 18.000,00$ & $\mathrm{R} \$ 3.600,00$ ano \\
\hline
\end{tabular}

\begin{tabular}{|c|c|c|c|}
\hline Material & $\begin{array}{c}\text { Valor } \\
\text { unitário }\end{array}$ & $\begin{array}{l}\text { Quantidade } \\
\text { por paciente }\end{array}$ & $\begin{array}{l}\text { Custo por } \\
\text { paciente }\end{array}$ \\
\hline Técnico & & & $\mathrm{R} \$ 10,0$ \\
\hline Bobina papel & $R \$ 15,00$ & $1 / 20$ & $R \$ \quad 0,75$ \\
\hline Total & & & $\mathrm{R} \$ 10,75$ \\
\hline
\end{tabular}

teríamos uma melhor capacidade para identificá-lo se estudássemos as subpopulações de células ganglionares mais susceptíveis, como é o caso dos cones azuis ${ }^{(19-2)}$ ou de células My por meio da tecnologia de freqüência duplicada ${ }^{(21)}$.

\section{CONCLUSÕES}

Podemos constatar que não houve diferença estatisticamente significante entre as medidas de MD no FDT antes e após o procedimento assim como para PSD. Apesar de termos usado a estratégia "screening" para realizar o primeiro exame em todos os pacientes (com o objetivo de diminuir a influência do efeito aprendizado) a média do MD pós-cirúrgica foi melhor do que a pré-cirúrgica, embora sem atingir significância estatística.

Apesar de não existirem estudos que comprovem a segurança do procedimento em pacientes com dano glaucomatoso, em que o aumento agudo da pressão intra-ocular, mesmo que súbito, possa ser deletério, o FDT, exame com alta sensibilidade e especificidade ${ }^{(16)}$ e de baixo custo, poderia ser utilizado como exame pré-operatório em pacientes submetidos à cirurgia refrativa (LASIK), de alto custo, com o propósito de evitar tal procedimento em pacientes com glaucoma.

\section{ABSTRACT}

Purpose: To check if there is functional damage to magnocellular cells using the frequency doubling technology (FDT), after laser assisted in situ keratomileusis-LASIK intraocular pressure increase and to analyze the benefit of this test in patients undergoing refractive surgery, regarding involved costs. Methods: Prospective study in 19 ametropic patients undergoing refractive laser assisted in situ keratomileusis surgery, at the "Hospital Oftalmológico de Sorocaba". Two frequency doubling technology examinations were performed in both eyes of patients before LASIK and one examination was done after the surgery. The assessment of the difference between the measurements of MD (Delta MD) was considered the dependent variable to formulate an evaluation of multiple regression. The costs of the frequency doubling technology and LASIK were analyzed. Results: There was no difference between the MD (mean deviation) and PSD (pattern standard deviation) before and after the procedure $(p=0.4454$ and $p=0.9716$, respectively). The continuous variables did not significantly influence Delta $\mathrm{MD}\left(\mathrm{r}^{2}=4.1 \% ; \mathrm{F}=0.15 ; \mathrm{p}=0.96\right)$. We considered 1500 LASIK surgeries during one year at the "Hospital Oftalmológico de Sorocaba", and the average cost of this surgery is R\$373.27. The frequency doubling technology costs R \$ 13.15 for each patient. Conclusions: There was no statistically significant difference between MD and PSD (pattern standard deviation) before and after the surgery. The frequency doubling technology has high sensitivity and specificity and low cost, so it could be used in patients undergoing refractive surgery (high cost) to avoid the laser assisted in situ keratomileusis in glaucoma patients.

Keywords: Laser assisted in situ keratomileusis; Cost -benefit analysis; Refractive errors/surgery; Retinal ganglion cells/ pathology; Perimetry/methods

\section{REFERÊNCIAS}

1. Sponsel WE, Arango S, Trigo Y, Mensah J. Clinical classification of glaucomatous visual field loss by frequency doubling perimetry. Am J Ophthalmol 1998;125:830-6.

2. Beck RW, Berstrom TJ, Lichter PR. A clinical comparison of visual testing with a new automated perimeter, the Humprey Field Analyzer and the Goldmann perimeter. Ophthalmology 1985;92:77-82

3. Katz J, Quigley HA, Sommer A. Repeatability of the glaucoma Hemifield test in Automated perimetry. Invest Ophthalmol Vis Sci 1995;36:1658-86.

4. McCrary JA, Feigon J, Computerized perimetry in neurophthalmology. Ophthalmology 1979;86:1287-301.

5. Johnson CA, Keltner JL, Balestrery FG. Suprathreshold static perimetry in glaucom and other optic nerve disease. Ophthalmology 1979;86:1278-86.

6. Quigley HA, Addicks EM, Green R. Optic nerve damage in human glaucoma. Arch Ophthalmol 1982;100:135-46.

7. Harwerth RS, Carter-Dawson L, Shen F, Smith EL, Crawford MLJ. Ganglion cell losses underlying visual field defects from experimental glaucoma. Ivest Ophthalmol Vis Sci 1999;40:2242-50.

8. Johnson CA, Wall M, Fingeret M, Lalle P. A primer for Frequency Doubling Technology. Dublin, CA, USA: Humphrey Systems.

9. Wilson SE. Lasik: management of commun complications. Cornea 1998;17: 459-67.

10. Bushley DM, Parmley DC, Paglen P. Visual field defect associated with laser in situ keratomileusis. Am J Ophthalmol 2000;129:668-71.

11. Cameron BD, Saffra NA, Strominger MB. Laser in situ keratomileusis induced optic neuropathy [commented on Ophthalmology 2002;109:818; discussion p.818-9]. Ophthalmology 2001;108:660-5.

12. Gürses- Özden R, Pons ME, Barbieri C, Ishikawa H, Buxton DF, Liebmann JM, et al. Scanning laser polarimetry measurements after laser-assisted in situ keratomileusis. Am J Ophthalmol 2000;129:461-4.

13. Choplin NT, Schlhorn SC. The effect of excimer laser photorefractive keratectomy for myopia on nerve fiber layer thickness measurements as determined by scanning laser polarimetry. Ophthalmology 1999;106:1019-23.

14. Gürses- Özden R, Liebmann JM, Buxton DF, Schuffner D, Soloway BD, Ritch R. Retinal nerve fiber layer thickness remains unchanged following laser-assisted in situ keratomileusis Am J Ophthalmol 2001;132:512-6.

15. Cello KE, Nelson-Quigg JM, Johnson CA. Frequency doubling technology perimetry for detection of glaucomatous visual field loss. Am J Ophthalmol 2000;129:314-22.

16. Patel SC, Friedman DS, Varadkar P, Robin AL. Algorithm for interpreting the results of frequency doubling perimetry. Am J Ophthalmol 2000;129:323-7. 
17. Falk JA. Gestão de custos para Hospitais São Paulo: Atlas 2001.

18. Tielsch JM, Katz J, Singh K, Quigley HA, Gottsch JD, Javitt J, et al. A population based evaluation of glaucoma screening. The Baltimore eye Survey. Am J Epidemiol 1991;134:1102-10.

19. Bowd C, Zangwill LM, Berry CC, Blumenthal EZ, Vasile C, Sanchez-Galeana $\mathrm{C}$, et al. Detecting early glaucoma by assesment of retinal nerve fiber layer thickness and visual function. Invest Ophthalmol Vis Sci 2001;42:1993-2003.
20. Landers J, Goldberg I, Grahan S. A comparison of short wavelenght automated perimetry with frequency doubling perimetry for the early detection of visual field loss in ocular hypertension. Clin Experiment Ophthalmol 2000; 28:248-52.

21. Quigley HA. Identification of glaucoma-related visual field abnormality with the screening protocol of frequency doubling perimetry. Am J Ophthalmol 1998;125:819-29.

\section{Congresso Internacional de Catarata e Cirurgia Refrativa}

Centro de Convenções de Pernambuco OLINDA - PE

\section{1 a 24 de abril de 2004}

\section{PROMOÇÃO:}

Sociedade Brasileira de Catarata e Implantes Intra-oculares

Sociedade Brasileira de Cirurgia Refrativa

\section{INFORMAÇÕES:}

Tel.: (81) 3442-1940 - Tel./fax: (81) 3265-7419

e-mails: sbcii@uol.com.br ou catarata@hotlink.com.br

home pages: www.catarata-refrativa.com.br e www.sbcii.com.br 